\title{
TeV particle direct detection in space - Recent results from the DAMPE mission
}

\author{
Giovanni Marsella on behalf of DAMPE Coll.* \\ $\dagger$ \\ Dipartimento di Matematica e Fisica "E. De Giorgi", Università del Salento and INFN, Lecce. \\ Italy \\ E-mail: giovanni.marella@le.infn.it
}

\begin{abstract}
Since its successful launch to a Low Earth Orbit in December 2015, the DAMPE (DArk Matter Particle Explorer) satellite mission has been preforming excellently, which allows the experiment to collect a large high quality data sample of high energy cosmic rays directly in space. With a relatively large acceptance, a thick BGO homogeneous calorimeter, and a precise silicon tracker, DAMPE is designed to measure multi-TeV particles in space with unprecedented precision. A first measurement of electron plus positron total flux up to $4.6 \mathrm{TeV}$ based on the first 18 months of data has been published. Other results, including proton and Helium fluxes up to $100 \mathrm{TeV} /$ nucleon, are becoming available. In this talk, the in-orbit performance of the DAMPE detector as well as the latest DAMPE data analysis results will be presented.
\end{abstract}

XXIX International Symposium on Lepton Photon Interactions at High Energies - LeptonPhoton2019 August 5-10, 2019

Toronto, Canada

\footnotetext{
* Speaker.

$\dagger$ The DAMPE mission is funded by the strategic priority science and technology projects in space science of Chinese Academy of Sciences. In China the data analysis is supported in part by the National Key Research and Development Program of China (No. 2016YFA0400200), the National Natural Science Foundation of China (Nos. 11525313, 11622327, 11722328, U1738205, U1738207, U1738208), the strategic priority science and technology projects of Chinese Academy of Sciences (No. XDA15051100), and the 100 Talents Program of Chinese Academy of Sciences. In Europe the activities and the data analysis are supported by the Swiss National Science Foundation (SNSF), Switzerland, and the National Institute for Nuclear Physics (INFN), Italy.
} 
Table 1: Summary of the design parameters and expected performance of DAMPE instrument

\begin{tabular}{ll}
\hline Parameter & Value \\
\hline Energy range of $\gamma$-rays/electrons & $5 \mathrm{GeV}-10 \mathrm{TeV}$ \\
Energy resolution of $\gamma$-rays/electrons & $1.5 \%$ at $800 \mathrm{GeV}$ \\
Energy range of protons/heavy nuclei & $50 \mathrm{GeV}-100 \mathrm{TeV}$ \\
Energy resolution of protons & $40 \%$ at $800 \mathrm{GeV}$ \\
Effective area at normal incidence $(\gamma$-rays) & $1100 \mathrm{~cm}^{2}$ at $100 \mathrm{GeV}$ \\
Geometric factor for electrons & $0.3 \mathrm{~m}^{2} \mathrm{sr} \mathrm{above} 30 \mathrm{GeV}$ \\
Photon angular resolution & $0.2^{\circ}$ at $100 \mathrm{GeV}$ \\
Field of View (FoV) & $1.0 \mathrm{sr}$ \\
\hline
\end{tabular}

\section{Introduction}

The DArk Matter Particle Explorer (DAMPE [1]), was successfully launched into a sunsynchronous orbit at the altitude of $500 \mathrm{~km}$ on December 17th 2015 from the Jiuquan launch base. DAMPE offers a new opportunity for advancing our knowledge of cosmic rays, dark matter, and gamma-ray astronomy. In this paper the recent scientific results are outlined and discussed. DAMPE is able to detect electrons/positrons, gamma rays, protons, helium nuclei and other heavy ions in a wide energy range with much improved energy resolution and large acceptance (see Table 1 for summary of the instrument parameters) than precedent detectors. There are 3 main scientific objectives addressed by DAMPE: (1) Gamma-ray astronomy; (2) indirect dark matter detection and (3) the cosmic ray physics.

\subsection{The detector}

The DAMPE detector is made by 4 main sub-detectors: a Plastic Scintillator strip Detector (PSD), a Silicon-Tungsten tracKer-converter (STK), a Bismute Germanate crystal (BGO) imaging calorimeter and a NeUtron Detector (NUD). The PSD is made of 82 bars of plastic scintillator in 2 planes with double layer configuration. It provides charged-particle background rejection for gamma rays (anti-coincidence detector) and measures the electric charge $\mathrm{z}$ of incident particles with $\mathrm{z}<28$. The STK is made of 6 planes with 2 single-sided silicon layers [2]. Three thin tungsten layers are added for gamma conversion to $e^{+} / e^{-}$. It measures the charges and the trajectories of charged particles, and allows to reconstruct the directions of incident photons. The track reconstruction has a spatial resolution better than $70 \mu \mathrm{m}$ for cosmic rays $\left(\Theta_{i n c}<60^{\circ}\right)$ and an angular resolution $\sim 0.2^{\circ}$ for gamma rays at $10 \mathrm{GeV}$. The hodoscopic BGO calorimeter, with a total depth of about 32 radiation lengths, allows to measure the energy of incident particles with high resolution and to provide efficient electron/hadron identification. It is made of 14 layers, each one made of 22 bars. Thanks to the multi-dynode readout design, the BGO calorimeter enables a measurement of the energy of electrons or $\gamma$-rays up to $10 \mathrm{TeV}$ without saturation. The energy deposited in the BGO calorimeter underestimates the true energy of incident particles. Electrons and photons can lose a significant fraction of their energy in the dead materials of calorimeter. The true energy of electrons and photons is evaluated by properly modelling the transversal and longitudinal development of electromagnetic showers in the calorimeter. The details of these procedures can be found in $[3,4]$. 

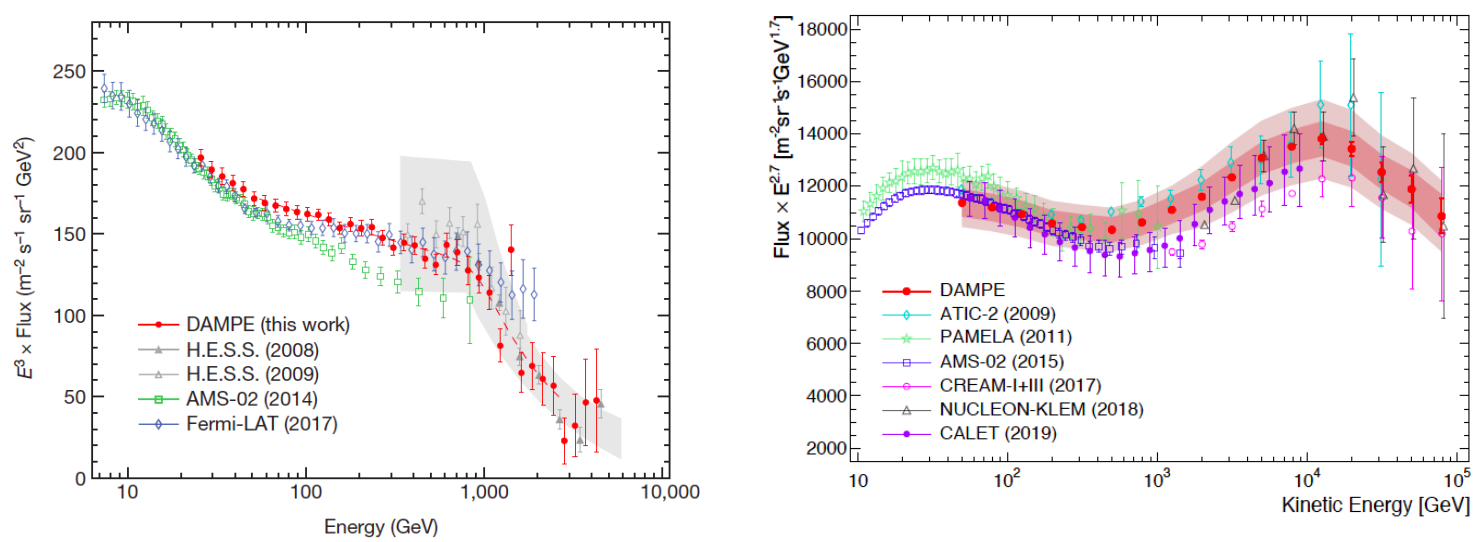

Figure 1: Left: The CRE spectrum (multiplied by $E^{3}$ ) measured by DAMPE. The red dashed line represents a smoothly broken power-law model that best fits the DAMPE data in the range $55 \mathrm{GeV}$ to $2.63 \mathrm{TeV}$. Also shown are the direct measurements from the space-borne experiments and the indirect measurement by the H.E.S.S. Collaboration (the grey band represents its systematic errors apart from the approximately $15 \%$ energy scale uncertainty). The error bars $( \pm 1 \sigma)$ include both systematic and statistical uncertainties added in quadrature. Right: The Proton spectrum (multiplied by $E^{2.7}$ ) measured by DAMPE. T(red points), compared with previous results by the principal direct measurement experiments.

The energy measurements for cosmic-ray protons and nuclei are much more complicated than that for electrons or gamma rays, as hadronic showers generally are not fully contained in the BGO. Moreover hadronic showers include an electromagnetic and a hadronic component with large eventby-event fluctuations, which brings relatively large uncertainties in the energy deposition. An unfolding algorithm based on the Bayes theorem has been implemented to estimate the primary energy spectra of cosmic-ray nuclei. DAMPE can measure hadronic cosmic rays up to an energy of $100 \mathrm{TeV}$ without significant saturation.

Finally, the NUD provides an independent measurement and further improvement of the electron/hadron identification. It is made of one layer of 4 boron-doped plastic scintillators. The detector is very stable since January the 1 st 2016 [5]. It is collecting data at a rate of 5 Mevents/day for a total 6.5 billion events in 3.5 years corresponding to 7 full scans of the sky. The exposure time is affected by the South Atlantic Anomaly $(\sim 4.5 \%)$, the on-orbit calibration $(\sim 1.5 \%)$ and instrumental dead time $(\sim 18.5 \%)$. In the following the principal Physics results are presented.

\subsection{Electrons and $\gamma$-rays}

The measurement of the total spectrum of cosmic ray electrons+positrons is the first important result of DAMPE thanks to a high identification and discrimination power of protons from electron/positrons. The algorithm for electron/proton identification is an image based pattern recognition method. MC simulation and beam test data showed that electrons and protons can be well separated. In the GeV-TeV energy range, the proton rejection power reaches a level of $10^{5}$, while keeping at least a 90\% electron identification efficiency. In addition, the High Energy trigger has been optimised to suppress the proton events by a factor of 3. The electron+positron spectrum obtained is show in fig. 1 (Left). The contamination of the proton background is estimated to be 

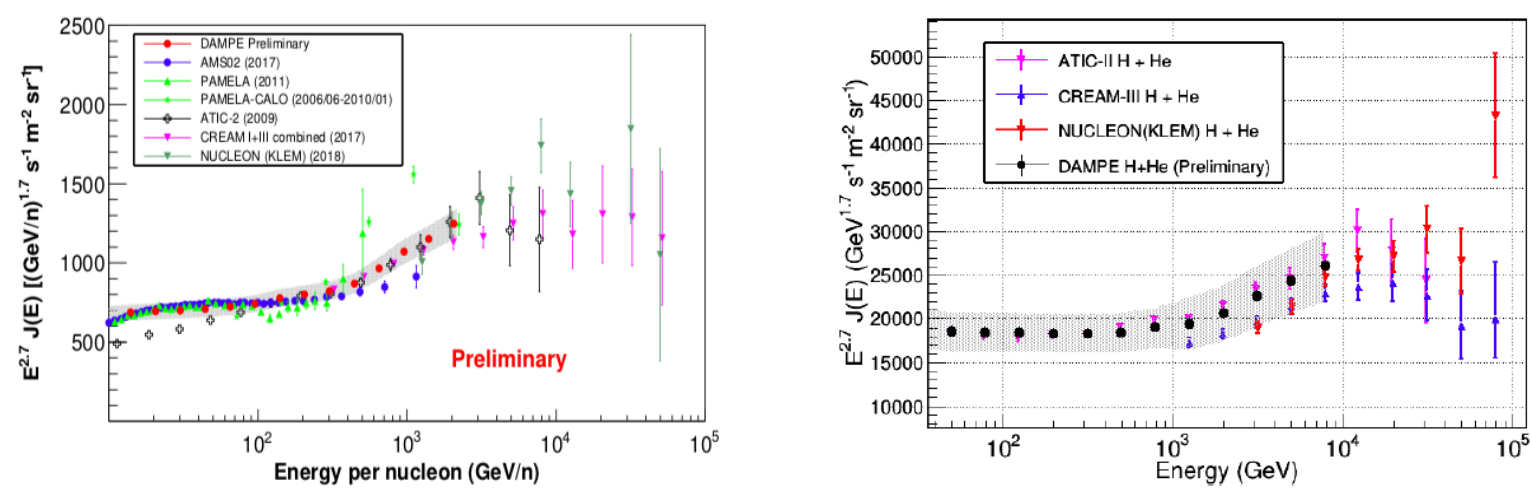

Figure 2: Left: Helium flux weighted with $E^{2.7}$ as a function of the primary energy per nucleon, compared with previous measurements performed by other experiments. The dashed grey area describes the systematic uncertianties. Right: The $\mathrm{H}+\mathrm{He}$ spectrum measured with DAMPE compared with other experiments

less than $3 \%$ in the energy range of $50 \mathrm{GeV}-1 \mathrm{TeV}$. All details on the analysis results and on the systematic uncertainties can be found in published paper [6]. Using the PSD as a veto photons have been selected, allowing the construction of a $\gamma$-rays sky map where the principal sources are evident [7]. Also the principal variable $\gamma$ sources have been observed and analysed. The latest results were presented at ICRC 2019 [8].

\subsection{Cosmic Rays}

In this section the latest results on CR physics obtained with DAMPE are presented. The PSD and the STK detectors allow a good particle selection. The proton spectrum in the energy range from $40 \mathrm{GeV}$ to $100 \mathrm{TeV}$ is shown in fig. 1 (Right). Seventeen months of DAMPE on-orbit data from January 1st, 2016 to June 30th, 2018 have been analysed. Error bars represent the $1 \sigma$ statistical uncertainties of the DAMPE measurements, and the shaded bands show the systematic uncertainties associated with the analysis procedure (inner band) and the total systematic uncertainties including those from the hadronic models (outer band) [4]. Previous measurements by principal space detectors are overlaid for comparison. The DAMPE proton spectrum is consistent with those of PAMELA and AMS-02. At higher energies, our results are also consistent with that of ATIC-2, CREAM, NUCLEON and CALET when the systematic uncertainties are taken into account. Fig. 2 (Left) shows the preliminary $E^{2.7}$ weighted Helium flux as a function of the primary energy per nucleon measured by DAMPE, compared with previous results of other space experiments [9]. A spectral hardening is clearly observed at energies of few hundreds of $\mathrm{GeV}$ per nucleon. Because of the calorimeter features (1.6 interaction lenghts) that allow to deposit only a small percentage of the total energy, an unfolding procedure [10] has been adopted in order to reconstruct the primary energy of Helium nuclei. The grey band describes the overall total systematic uncertainty on the flux. Fig. 2 (Right) shows the preliminary $\mathrm{H}+\mathrm{He}$ spectrum[11] measured with DAMPE using $3.8 \times 10^{7} \mathrm{CR}$ events collected from the 1 st January 2016 up to the 30th March 2019. The total systematic uncertainty in the flux is $12 \%$ below $1 \mathrm{TeV}$ and $16 \%$ above $1 \mathrm{TeV}$, as shown by the grey shaded area. The DAMPE H + He spectrum is compared with the ATIC-02, CREAM-III, and 
NUCLEON. Considering the systematic uncertainties, the spectrum is compatible with the previous direct-detection experiments. A clear spectral hardening can be observed below $1 \mathrm{TeV}$ (energy per nucleus), which is in agreement with previously observed hardening at $250 \mathrm{GeV} / \mathrm{n}$, measured by other experiments separately for $\mathrm{H}, \mathrm{He}$ and heavier cosmic ray nuclei. The extension of the measurement up to $100 \mathrm{TeV}$ is currently ongoing.

\section{Conclusions}

The DAMPE detector is taking data in a stable condition since January 2016 and the first results have been presented. The spectral hardening and softening of proton spectrum and the hardening of helium spectrum are not compatible with the paradigm of a unique power law spectrum up to the all-particle knee at PeV energies, thus implying a deep revision of CR modelling in the Galaxy. Therefore, the current DAMPE measurement of the proton and helium spectrum, together with other measurements from the space and ground based experiments, puts a severe constraint on the models of Galactic CRs, which suggests some hints for a better understanding of CRs origin, propagation and acceleration mechanisms. In the future, with the increasing of the data collection and improving of the analysis procedures, DAMPE is expected to provide important measurements of the Cosmic Rays spectrum up to $100 \mathrm{TeV}$ particle energy, and of the $\gamma$-ray emission from Galactic and extragalactic sources. This will contribute to a better understanding of the origin and propagation mechanism of high energy Cosmic Rays and the possibility of probing the nature of dark matter.

\section{References}

[1] J. Chang et al., “The DArk Matter Particle Explorer mission”, Astrop. Physics 95 (2017) 6-24.

[2] T. Dong et al., "Charge measurement of cosmic ray nuclei with the plastic scintillator detector of DAMPE", Astrop. Physics 105 (2019) 31 [arXiv:1810.10784].

[3] C. Yue et al., "A Parameterized Energy Correction Method for Electromagnetic Showers in BGO-ECAL of DAMPE” Nucl. Instrum. Methods A , 856 (2017) 11.

[4] "Q. An et al. [DAMPE collaboration], "Measurement of the cosmic-ray proton spectrum from $40 \mathrm{GeV}$ to $100 \mathrm{TeV}$ with the DAMPE satellite", Sci. Adv. 5, eaax3793 (2019)"..

[5] G. Ambrosi et al. [DAMPE Collaboration], "The on-orbit calibration of DArk Matter Particle Explorer", Astrop. Physics 106 (2019) 18.

[6] G. Ambrosi et al., "Direct detection of TeV break in the cosmic ray $e^{-}+e^{+}$spectrum", Nature, $\mathbf{5 5 2}$, (2017) 63-66

[7] X. Li et al., “Recent Gamma-ray Results from DAMPE”, Proc. Sci. ICRC2019 (2019) 576.

[8] M. Munoz et al., “Gamma-ray pulsars with DAMPE”, Proc. Sci. ICRC2019 (2019) 587

[9] M. Di Santo et al., "Helium spectrum in the cosmic rays measured by the DAMPE detector", Proc. Sci. ICRC2019 (2019) 058

[10] G. D?Agostini, "A multidimensional unfolding method based on Bayes? theorem", Nuclear Instruments and Methods in Physics Research A 362 (1995) 362.

[11] I. DeMitri et al., "Measurement of the cosmic-ray proton + helium spectrum with DAMPE", Proc. Sci. ICRC2019 (2019) 148. 